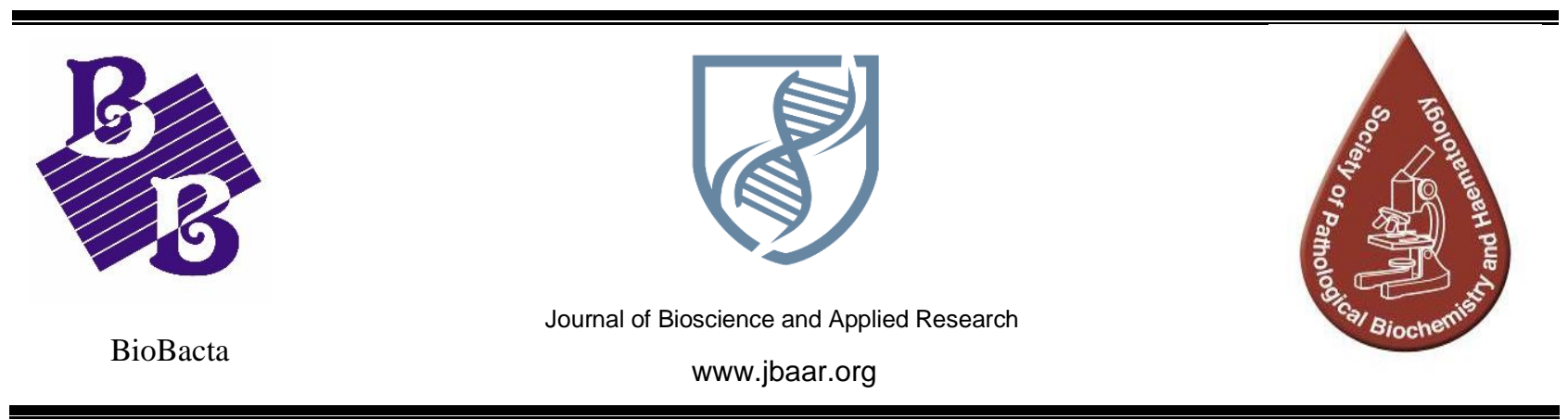

\title{
Elevated serum NAGAL levels were associated with cardiovascular diseases in
} pediatric chronic kidney disease

\author{
Mohamed A. Abdelrazek ${ }^{1,2 *}$, Hossam T. ElAgamy ${ }^{3}$, Lamiaa A. Barakat ${ }^{3}$, Gamal T. Soliman ${ }^{4}$, \\ Mohamed A. Basuni 5 \\ ${ }^{1}$ Biotechnology Research Center, New Damietta, Egypt \\ ${ }^{2}$ Sherbin Central Hospital, Ad Daqahliyah, Ministry of Health, Egypt \\ ${ }^{3}$ Faculty of Science, Port-Said University, Port-Said, Egypt \\ ${ }^{4}$ Faculty of Medicine, El-Minia University, El-Minia, Egypt \\ ${ }^{5}$ Mansoura Children hospital, Mansoura University, Mansoura, Egypt \\ * Corresponding author: Mohamed A. Abdelrazek, PhD \\ Biotechnology Research Center, 23 July St., Industrial Zone, New Damietta 34517, Egypt.
}

Tel: 02/ 01007036126; E-mail: maabdelrazek@yahoo.com

Received 24/2/2020; Accepted 21/3/2020

\section{Abstract}

Background: Early cardiovascular disease (CVD) management and prediction have become mandatory in children with chronic kidney disease (CKD). Association between neutrophil gelatinase-associated lipocalin (NGAL) and CVD events in pediatric CKD patients remains unclear. Thus, we aimed to evaluate this relationship and to clarify the association between NGAL serum levels and some CVD related parameters. Subjects and methods: A total of 70 children patients with CKD (30 with and 40 without CVD). The patient's data were retrospectively recorded from the medical files of each patient. NGAL serum levels were measured by ELISA commercial kits. Association between different parameters was assessed by the Pearson correlation coefficient. Results: NGAL serum levels were significantly $(P<0.0001)$ higher in patients with CVD (2450 (1335-2880) $\mathrm{pg} / \mathrm{mL}$ ) than patients without CVD (371 (285-1363) pg/mL) and healthy controls (295 (166-357) pg/mL). At 1300 $\mathrm{pg} / \mathrm{mL}, \mathrm{NGAL}$ has a good CVD predictive function with a high area under curve (AUC=0.871). The sensitivity, specificity, positive predictive value, negative predictive value, and accuracy were 80, 80, 63.2, 90.3, and 80\%, respectively. CVD risk increase with elevated NGAL serum levels (>1300 pg/mL) (OR=2.57, 95 CI (1.62-4.09), $P<0.0001)$. Carotid intima thickness was associated with elevated NGAL levels. Both NGAL and carotid intima thickness were significantly correlated with dialysis duration, uric acid, and lipid profile. Conclusion: NGAL was associated with CVD events in children with CKD with good predictive value supporting NGAL putative role in CVD pathophysiology. But NGAL in CVD is still in the early stages and future studied needed to evaluate its association with CVD severity.

Keywords: Neutrophil gelatinase-associated lipocalin, NGAL, cardiovascular disease, chronic kidney disease. DOI: 10.21608/jbaar.2020.116101 


\section{Introduction}

In pediatric patients with chronic kidney disease (CKD), cardiovascular disease (CVD) is a leading cause of death (do Val et al., 2019). It is not surprising that the guidelines of the American Heart Association for the reduction of CVD risk in high-risk pediatric patients stratified pediatric CKD in the highest risk category for CVD development (Mitsnefes, 2012). Thus, early CVD prediction and management have become mandatory in children and adolescents with CKD (El-Gamasy and Mawlana, 2019). Moreover, the accurate CVD risk assessment at an early stage would facilitate more focused and aggressive treatment of patients with the goal to decrease event rates (D'Marco et al., 2015).

Many traditional risk factors related to renal function loss (anemia, bone mineral disorders, inflammation, and oxidative stress) are also related to high CVD incidence in CKD patients. Whether biomarkers in CKD patients has been at the core of extensive research to help improve patients at CVD risk identification (D'Marco et al., 2015; Niizuma et al., 2017). Because of various damaging stimuli, neutrophil gelatinaseassociated lipocalin (NGAL) is released from injured renal tubular cells. It is known to be one of the most promising biomarkers of impending kidney injury (Devarajan, 2010). As well as renal tubules, the vascular walls in rats responded to various types of damage by overexpressing NGAL protein (Cruz et al., 2012). Evidence suggests that NGAL plays a crucial role in atherosclerosis in plaque instability and vascular remodeling (Hemdahl et al., 2006; Folkesson et al., 2007). Increased NGAL levels have been reported in many CVD conditions, including coronary heart disease, both acute and chronic heart failure and stroke (Cruz et al., 2012). Collectively, these findings provide biological plausibility for NGAL potential role

DOI: 10.21608/jbaar.2020.116101 as CVD biomarker in patients with CKD (Cruz et al., 2012).

To date, the relationship between NGAL and CVD events in pediatric CKD patients remains unclear. We, therefore, aimed to evaluate this relationship and to clarify the association between NGAL serum levels and some parameters related to CVD events including dialysis duration, lipid profile and carotid intima-media thickness.

\section{Material and methods}

\subsection{Study population}

The present study consisting of children $(n=70)$ aged 416 years with CKD (stage 5) maintained on dialysis in the Pediatric Nephrology Unit of Mansoura Children's Hospital, Mansoura University, Mansoura, Egypt. CKD were urological abnormalities $(n=35)$, glomerulonephritis $(n=10)$, pyelonephritis $(n=14)$ and other unknown causes $(n=11)$. The patient's data were retrospectively recorded from the medical files of each patient. There were classified to 40 patients without and 30 with CVD events. All CVD events were confirmed through the participant's medical records. In addition, thirty control subjects were outpatients of the Mansoura Children's Hospital, aged 5-17 years, without any chronic diseases or renal impairment. Informed consent was obtained from the parents of each patient. This study has been performed according to the Helsinki Declaration's ethical guidelines.

\subsection{Laboratory and biomarker measurements}

Blood samples collected for serum NGAL analysis were centrifuged at $1000 \times \mathrm{g}$ for 20 minutes and stored at $-20^{\circ} \mathrm{C}$ until analysis. Using fresh serum, liver and kidney function tests and lipid profile were all measured on fully automated chemistry analyzer (DIALAB Autolyser 100, Neudorf, Austria) and minerals were determined using blood gas/electrolyte analyzer (Cobas b 121, Roche Diagnostics). NGAL 
was measured using ELISA commercial Kits (Boster Biological Technology, Pleasanton, California, USA).

\subsection{Statistical analysis}

GraphPad Prism and SPSS software were used for data analysis. Non-normally distributed data are shown as median (interquartile range), and normally distributed data are shown as mean \pm standard deviation (SD). Univariate analysis of variance (ANOVA) and the Kruskal-Wallis test was used to compare values between groups with normal and skewed variable distribution, respectively. Proportions in groups were compared using the Pearson $\chi 2$ test. $P<0.05$ was considered statistically significant. The Receiveroperating characteristic (ROC) curve was used to evaluate the predictive function of NGAL serum levels for identifying new-onset CVD in hemodialysis children patients. To assess whether any two variables are associated, the Pearson correlation analysis was used.

\section{Results}

\subsection{Characteristics of study populations}

Table 1 showed the main demographic and clinical data of hemodialysis children patients with and without CVD and healthy children controls. Patients with CVD were older $(P=0.0321)$ than others. CKD patients (with/without CVD) were associated with significantly elevated serum levels of creatinine, urea, blood urea nitrogen, uric acid, alkaline phosphatase, sodium, potassium, and phosphorus. There was a significant difference in dialysis duration and carotid intima thickness between children patients with CVD events and other patients without CVD and healthy controls. Also, children patients with CVD events were associated with dyslipidemia, elevated serum levels of cholesterol, triglycerides, low-density lipoprotein, and very high-density lipoprotein and decreased serum levels of high-density lipoprotein.

\subsection{Elevated NGAL levels and CVD risk}

Median serum levels of NGAL were significantly $(P<0.0001)$ higher in patients with CVD (2450 (13352880) pg/mL) than who without CVD (371 (285-1363) $\mathrm{pg} / \mathrm{mL}$ ) and healthy controls (295 (166-357) pg/mL) (Figure 1). At 1300 pg/mL, NGAL has a good CVD predictive function with a high area under curve (AUC=0.871; Figure 2). The sensitivity, specificity, positive predictive value, negative predictive value, and accuracy were 80, 80, 63.2, 90.3 and $80 \%$, respectively. This power increased when patients with CVD were discriminated against from healthy controls only (Figure 3) and the mentioned values increased to $80,100,100,83.3$ and $90 \%$, respectively. The odds ratio $(\mathrm{OR}=2.57,95 \mathrm{CI}(1.62-4.09), P<0.0001)$ for $\mathrm{CVD}$ prevalence increased with the increase in NGAL serum levels (>1300 pg/mL).

\subsection{Association between NGAL, carotid intima thickness and other parameters}

Carotid intima thickness was associated with elevated NGAL levels. Both NGAL and carotid intima thickness were significantly correlated with dialysis duration and serum levels of uric acid, cholesterol, HDL and LDL (Tables 2 and 3). 
Table 1. Patients characteristics

\begin{tabular}{|c|c|c|c|c|}
\hline \multirow{2}{*}{ Parameter } & \multirow{2}{*}{ Healthy } & \multicolumn{2}{|c|}{ Hemodialysis patients } & \multirow{2}{*}{$P$ value } \\
\hline & & Without CVD & With CVD & \\
\hline Age (years) & $6.9 \pm 2.7$ & $5.9 \pm 3.1$ & $8.6 \pm 4.8$ & 0.0321 \\
\hline Gender (male/female) & $19 / 11$ & $58 / 27$ & $39 / 26$ & 0.579 \\
\hline Creatinine (mg/dL) & $0.55 \pm 0.1$ & $7.42 \pm 3.3$ & $6.67 \pm 1.8$ & $<0.0001$ \\
\hline Urea $(\mathrm{mg} / \mathrm{dL})$ & $18.6 \pm 3.4$ & $132.6 \pm 71.7$ & $90.5 \pm 50.2$ & $<0.0001$ \\
\hline BUN (mg/dL) & $9.4 \pm 2.64$ & $70.8 \pm 26.1$ & $72.6 \pm 25.9$ & $<0.0001$ \\
\hline Uric acid $(\mathrm{mg} / \mathrm{dL})$ & $3.2 \pm 0.49$ & $5.4 \pm 1.47$ & $6.6 \pm 1.34$ & $<0.0001$ \\
\hline ALT (U/L) & $20.1 \pm 1.4$ & $23.3 \pm 5.5$ & $22.3 \pm 4.99$ & 0.104 \\
\hline AST (U/L) & $17.2 \pm 3.3$ & $19.2 \pm 4.9$ & $18.0 \pm 2.9$ & 0.445 \\
\hline Albumin (g/dL) & $4.3 \pm 0.53$ & $3.88 \pm 0.27$ & $3.86 \pm 0.27$ & 0.002 \\
\hline Bilirubin (mg/dL) & $0.56 \pm 0.1$ & $0.57 \pm 0.05$ & $0.56 \pm 0.15$ & 0.942 \\
\hline Alkaline phosphatase (U/L) & $122(71-203)$ & $460(184-1524)$ & $626(269-706)$ & 0.0007 \\
\hline Sodium (mmol/L) & $131.7 \pm 1.58$ & $137.9 \pm 3.65$ & $138.3 \pm 3.63$ & $<0.0001$ \\
\hline Potassium (mmol/L) & $3.95 \pm 0.56$ & $4.76 \pm 1.18$ & $4.76 \pm 1.40$ & 0.0914 \\
\hline Calcium (mg/dL) & $9.28 \pm 0.38$ & $8.34 \pm 1.47$ & $8.27 \pm 1.36$ & 0.0222 \\
\hline Phosphorus (mg/dL) & $4.18 \pm 0.36$ & $5.25 \pm 1.17$ & $5.38 \pm 1.27$ & 0.0043 \\
\hline Dialysis duration (months) & - & $18.56 \pm 5.64$ & $30.52 \pm 6.06$ & $<0.0001$ \\
\hline Carotid intima thickness (mm) & $0.440 \pm 0.011$ & $0.449 \pm 0.005$ & $0.462 \pm 0.003$ & $<0.0001$ \\
\hline Cholesterol (mg/dL) & $111.6 \pm 23.4$ & $163.3 \pm 46.0$ & $197.4 \pm 60.4$ & $<0.0001$ \\
\hline Triglycerides (mg/dL) & $98(55-122)$ & $127(96-159)$ & $136(109-178)$ & 0.0485 \\
\hline HDL (mg/dL) & $58(48-69)$ & $55(39-76)$ & $15(8-27)$ & $<0.0001$ \\
\hline LDL (mg/dL) & $38(18-50)$ & $60(26-106)$ & $106(92-182)$ & $<0.0001$ \\
\hline vLDL (mg/dL) & $20(11-24)$ & $25(19-31)$ & $30(24-47)$ & 0.0031 \\
\hline
\end{tabular}

Abbreviations: $\mathrm{BUN}=$ blood urea nitrogen; $\mathrm{ALT}=$ Alanine aminotransferase $; \mathrm{AST}=\mathrm{Aspartate}$ aminotransferase $; \mathrm{HDL}=\mathrm{high}$ density lipoprotein; $\mathrm{LDL}=$ low density lipoprotein; $\mathrm{vLDL}=$ very high-density lipoprotein. Normally distributed variables were expressed as mean \pm SD. Non-normally distributed variables were expressed as median (interquartile range). Significant difference was determined using $X^{2}$ test, ANOVA and Kruskal-Wallis tests as appropriate. $P<0.05$ was considered significant. 


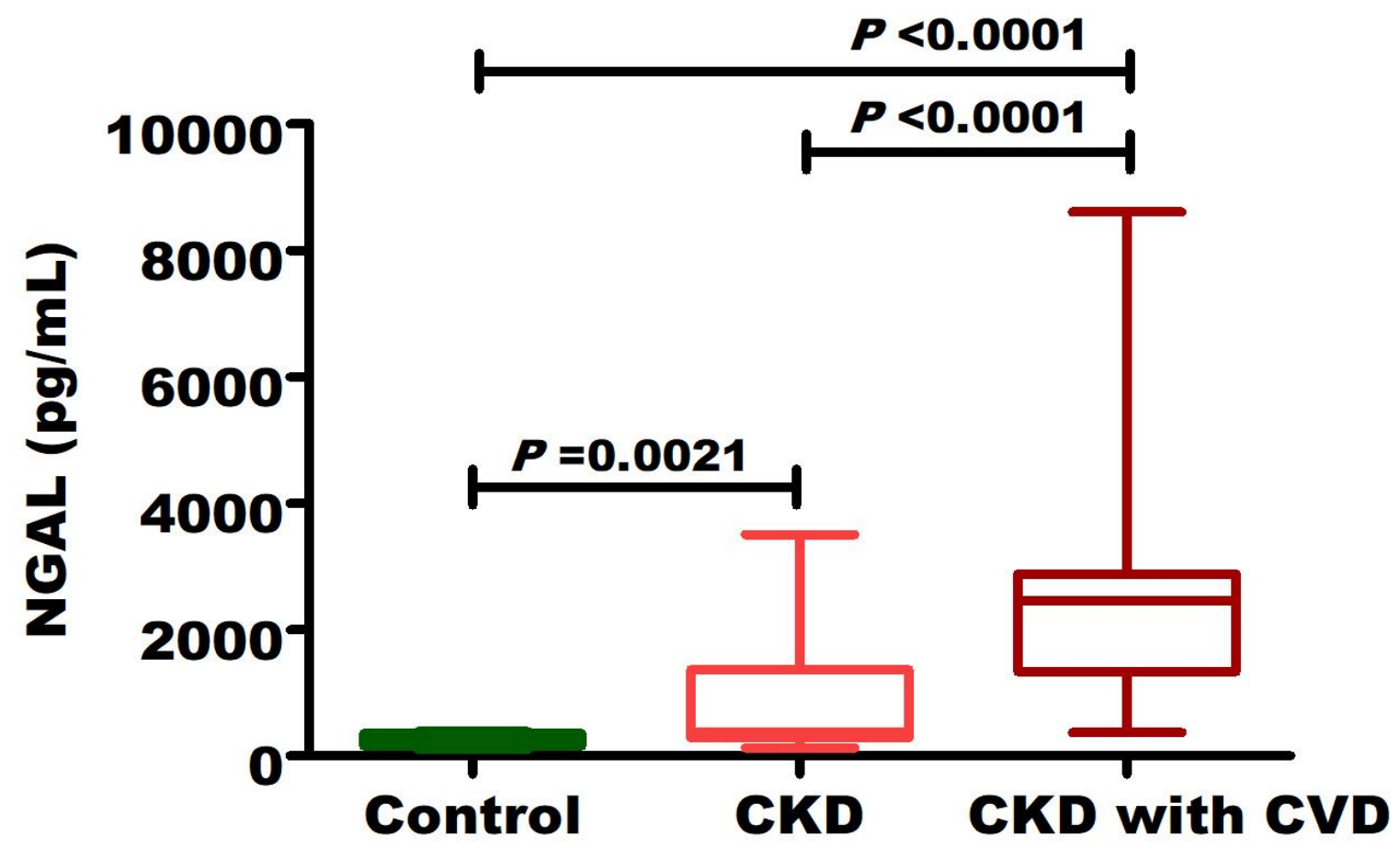

Figure 1. NGAL serum levels of included patients and controls. Significant difference was determined using Kruskal-Wallis tests and LSD as post-hoc comparison method.

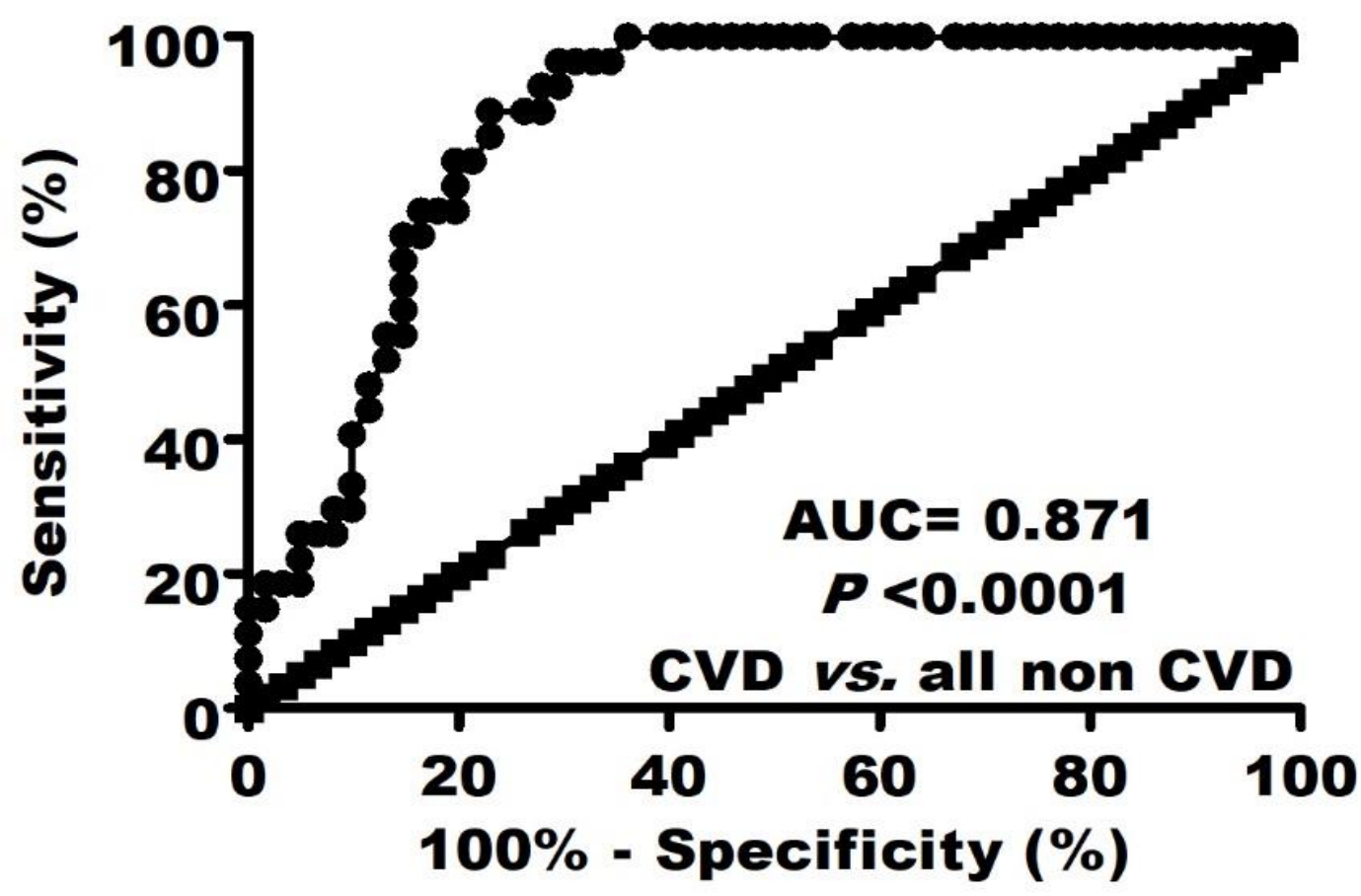

Figure 2. ROC analysis for NGAL to discriminate patients with CVD from all non-CVD individuals. 


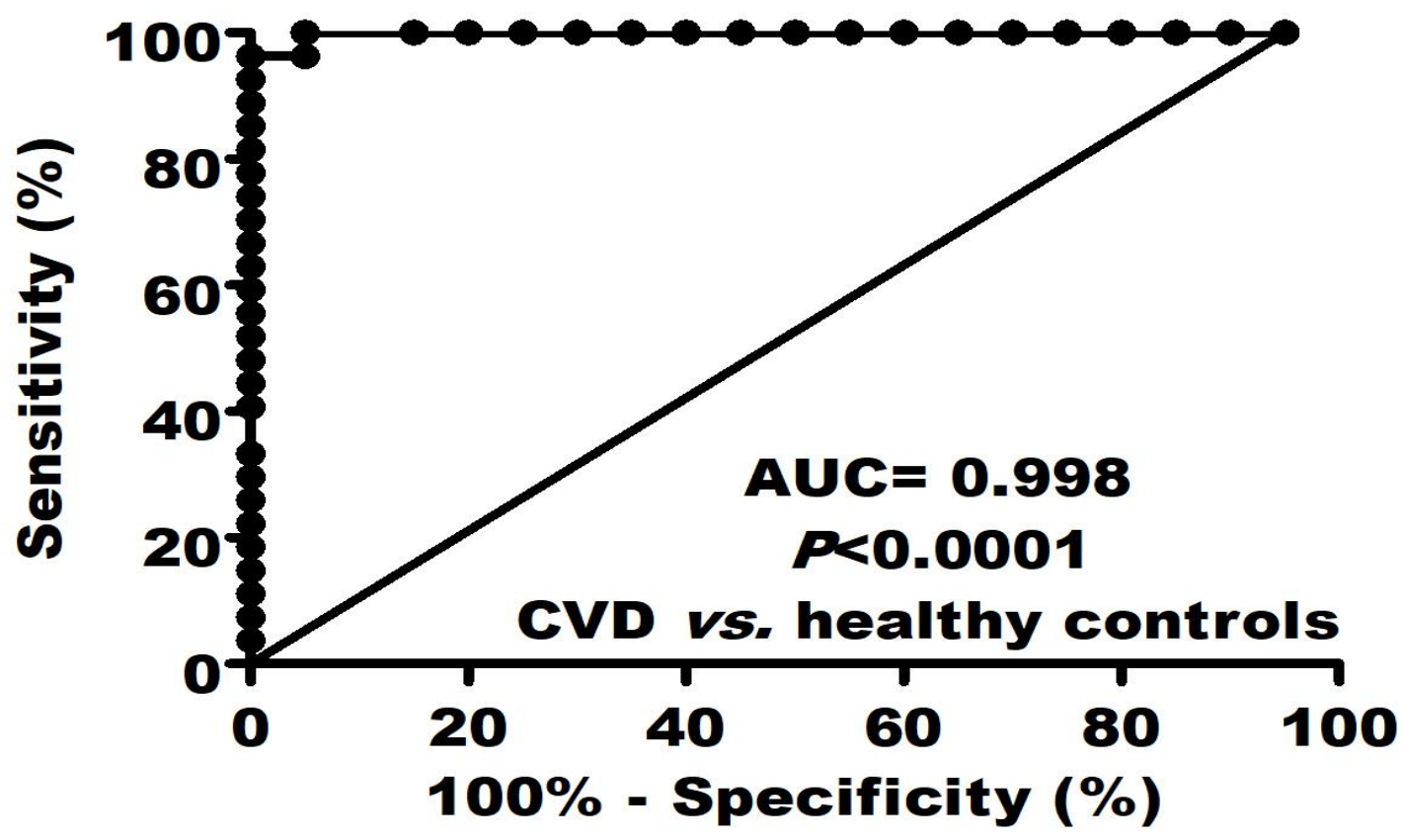

Figure 3. ROC analysis for NGAL to discriminate patients with CVD from healthy controls.

Table 2. Correlation between carotid thickness and other clinical parameters

\begin{tabular}{|l|c|c|}
\hline \multicolumn{1}{|c|}{$\begin{array}{c}\text { Factor correlated } \\
\text { with carotid thickness }\end{array}$} & $\begin{array}{c}\text { Pearson correlation } \\
\text { coefficient }(r)\end{array}$ & $P$ value \\
\hline Creatinine & 0.117 & 0.604 \\
\hline Urea & -0.04 & 0.889 \\
\hline Blood urea nitrogen & -0.01 & 0.973 \\
\hline Uric acid & 0.663 & $<0.001$ \\
\hline Creatinine & 0.117 & 0.604 \\
\hline ALT & -0.173 & 0.523 \\
\hline AST & -0.171 & 0.528 \\
\hline Albumin & 0.07 & 0.673 \\
\hline Bilirubin & -0.03 & 0.936 \\
\hline Alkaline phosphatase & 0.238 & 0.326 \\
\hline Sodium & 0.116 & 0.616 \\
\hline Potassium & 0.08 & 0.748 \\
\hline Calcium & 0.07 & 0.775 \\
\hline Phosphorus & 0.135 & 0.606 \\
\hline Dialysis duration & 0.696 & $<0.001$ \\
\hline Cholesterol & 0.328 & 0.006 \\
\hline Triglycerides & 0.08 & 0.527 \\
\hline HDL & -0.669 & $<0.001$ \\
\hline LDL & 0.423 & $<0.001$ \\
\hline vLDL & 0.214 & 0.082 \\
\hline NGAL & 0.546 & $<0.001$ \\
\hline & & \\
\hline & & \\
\hline
\end{tabular}

Abbreviations: $\mathrm{ALT}=$ Alanine aminotransferase; $\mathrm{AST}=$ Aspartate aminotransferase $\mathrm{HDL}=$ high density lipoprotein $; \mathrm{LDL}=$ low density lipoprotein; vLDL= very high-density lipoprotein. 
Table 3. Correlation between NGAL serum levels and other clinical parameters

\begin{tabular}{|l|c|c|}
\hline \multicolumn{1}{|c|}{$\begin{array}{c}\text { Factor correlated } \\
\text { with NGAL serum levels }\end{array}$} & $\begin{array}{c}\text { Pearson correlation } \\
\text { coefficient }(r)\end{array}$ & $P$ value \\
\hline Creatinine & -0.193 & 0.389 \\
\hline Urea & 0.016 & 0.955 \\
\hline Blood urea nitrogen & 0.135 & 0.632 \\
\hline Uric acid & 0.237 & 0.302 \\
\hline Creatinine & 0.117 & 0.604 \\
\hline ALT & -0.231 & 0.389 \\
\hline AST & -0.347 & 0.188 \\
\hline Albumin & -0.081 & 0.595 \\
\hline Bilirubin & 0.105 & 0.746 \\
\hline Alkaline phosphatase & -0.019 & 0.939 \\
\hline Sodium & -0.162 & 0.483 \\
\hline Potassium & -0.194 & 0.389 \\
\hline Calcium & -0.026 & 0.911 \\
\hline Phosphorus & -0.112 & 0.668 \\
\hline Carotid thickness & 0.546 & $<0.001$ \\
\hline Dialysis duration & 0.437 & $<0.001$ \\
\hline Cholesterol & 0.201 & 0.100 \\
\hline Triglycerides & 0.063 & 0.620 \\
\hline HDL & -0.489 & $<0.001$ \\
\hline LDL & 0.285 & 0.033 \\
\hline vLDL & 0.121 & 0.330 \\
\hline
\end{tabular}

Abbreviations: $\mathrm{ALT}=$ Alanine aminotransferase; $\mathrm{AST}=$ Aspartate aminotransferase $\mathrm{HDL}=$ high density lipoprotein; $\mathrm{LDL}=$ low density lipoprotein; vLDL= very high-density lipoprotein.

\section{Discussion}

Beyond the glomerular filtration rate (GFR), some studies clearly indicated that NGAL on its own represents a CKD progression marker (Bolignano et al., 2009b). Moreover, beyond the renal physiopathology boundaries, findings made in other studies indicated that this protein may be used in patients with CVD events (Bolignano et al., 2010). NGAL was reported to be independently associated with a higher risk of CVD events in adult patients with CKD. But they recommended further work to determine NGAL utility to improve risk prediction for CVD adverse outcomes (Zylka et al., 2016; Park et al., 2017). As in adults, CKD children patients have a high prevalence of uremia-related and traditional CVD risk factors (Mitsnefes, 2012). In this population, to our knowledge, NGAL did not evaluate as an early marker of CVD.

Here, NGAL serum levels were significantly higher in patients with CVD than patients without CVD and healthy controls. It has good CVD predictive power with good sensitivity, specificity, positive predictive value, negative predictive value and accuracy of 80,80 , $63.2,90.3$ and $80 \%$, respectively. Findings from human tissue and animal studies demonstrate that NGAL is highly expressed in the heart and also expressed in 
atherosclerotic plaques and both in failing myocarditis and myocardium (Cruz et al., 2012).

Compared to normal mice, aortas from atherosclerotic mice exhibited higher levels of NGAL mRNA. In the aorta of atherosclerotic mice, hypoxic stress increased NGAL mRNA expression, suggesting that NGAL expression was necessary for the development of myocardial infarction or possibly that NGAL was upregulated as infarction result. In contrast to normal aortas of control mice, in atherosclerotic mice abundant NGAL protein was seen in aortic plaques border regions and lipid core, and co-localized with matrix metalloproteinase (MMP)-9 (Hemdahl et al., 2006). In the acute heart failure rat model, the left coronary artery was ligated (Yndestad et al., 2009), causing transmural infarction of the left ventricular free wall. Rats with heart failure had significantly raised NGAL expression in the left ventricular non-ischemic area, representing development from acute to a chronic stage in this model (Yndestad et al., 2009). In an autoimmune myocarditis rat model, NGAL was strongly expressed in fibroblasts, vascular wall cells, and cardiomyocytes (Ding et al., 2010). In response to IL-1 $\beta$ stimulation, NGAL mRNA and protein expression in vascular smooth muscle cells were also upregulated in an NF- $\kappa \mathrm{B}$-dependent manner (te Boekhorst et al., 2011).

Compared to control (non-failing) human left ventricular tissue, tissue aliquots from failing myocardium (explantation of hearts from end-stage heart failure patients undergoing cardiac transplantation) exhibited strong NGAL immunostaining, with some immunoreactivity also seen in endothelial cells and vascular smooth muscle (Yndestad et al., 2009). Also, NGAL was detected in leukocytes, fibroblastoid cells, vascular wall cells and cardiomyocytes in human hearts with myocarditis (Ding et al., 2010).
Moreover, many clinical studies reported the role of NGAL in different CVD events. Compared with control subjects, chronic heart failure patients have been reported to have significantly elevated levels of both urine and serum NGAL (Damman et al., 2008; Bolignano et al., 2009a; Poniatowski et al., 2009; Yndestad et al., 2009). Both serum and urine NGAL levels correlated with various renal function indices including GFR, urinary albumin, creatinine, blood urea nitrogen and cystatin C (Shrestha et al., 2011). Similar to findings seen in chronic heart failure, serum NGAL also appeared to correlate with renal functions in acute heart failure (Aghel et al., 2010; Maisel et al., 2011) and coronary heart disease (Choi et al., 2008; Zografos et al., 2009).

In this study, carotid intima thickness was associated with elevated NGAL levels and both of them were significantly correlated with some CVD related risks such as dialysis duration and elevated serum levels of uric acid, cholesterol, HDL and LDL. In the cerebrovascular disease rats' model, NGAL was highly induced in the intima after balloon injury to the common carotid artery (Bu et al., 2006). NGAL was also reported to be significantly correlated to dyslipidemia (Dolapoglu and Beketaev, 2015) and uric acid (Tomczak et al., 2013).

\section{Conclusion}

NGAL was reported to be associated with CVD events in children with CKD. It has a good predictive value supporting NGAL putative role in CVD pathophysiology. But NGAL in CVD is still in the early stages and future studied needed to evaluate NGAL association with the severity of CVD.

\section{REFERENCES}

Aghel, A., Shrestha, K., Mullens, W., Borowski, A., and Tang, W. H. (2010). Serum neutrophil gelatinaseassociated lipocalin (NGAL) in predicting worsening renal function in acute decompensated heart failure. Journal of cardiac failure $16,49-54$. 
Bolignano, D., Basile, G., Parisi, P., Coppolino, G., Nicocia, G., and Buemi, M. (2009a). Increased plasma neutrophil gelatinase-associated lipocalin levels predict mortality in elderly patients with chronic heart failure. Rejuvenation research 12, 7-14.

Bolignano, D., Coppolino, G., Lacquaniti, A., and Buemi, M. (2010). From kidney to cardiovascular diseases: NGAL as a biomarker beyond the confines of nephrology. European journal of clinical investigation 40, 273-276.

Bolignano, D., Lacquaniti, A., Coppolino, G., Donato, V., Campo, S., Fazio, M. R., Nicocia, G., and Buemi, M. (2009b). Neutrophil gelatinaseassociated lipocalin (NGAL) and progression of chronic kidney disease. Clin J Am Soc Nephrol 4, 337 344.

Bu, D. X., Hemdahl, A. L., Gabrielsen, A., Fuxe, J., Zhu, C., Eriksson, P., and Yan, Z. Q. (2006). Induction of neutrophil gelatinase-associated lipocalin in vascular injury via activation of nuclear factorkappaB. The American journal of pathology 169, 2245 2253.

Choi, K. M., Lee, J. S., Kim, E. J., Baik, S. H., Seo, H. S., Choi, D. S., Oh, D. J., and Park, C. G. (2008). Implication of lipocalin-2 and visfatin levels in patients with coronary heart disease. European journal of endocrinology 158, 203-207.

Cruz, D. N., Gaiao, S., Maisel, A., Ronco, C., and Devarajan, P. (2012). Neutrophil gelatinaseassociated lipocalin as a biomarker of cardiovascular disease: a systematic review. Clin Chem Lab Med 50, 1533-1545.

D'Marco, L., Bellasi, A., and Raggi, P. (2015). Cardiovascular biomarkers in chronic kidney disease: state of current research and clinical applicability. Disease markers 2015, 586569.

Damman, K., van Veldhuisen, D. J., Navis, G., Voors, A. A., and Hillege, H. L. (2008). Urinary neutrophil gelatinase associated lipocalin (NGAL), a marker of tubular damage, is increased in patients with chronic heart failure. European journal of heart failure 10, 997-1000.

Devarajan, P. (2010). Neutrophil gelatinaseassociated lipocalin: a promising biomarker for human acute kidney injury. Biomark Med 4, 265-280.

Ding, L., Hanawa, H., Ota, Y., Hasegawa, G., Hao, K., Asami, F., Watanabe, R., Yoshida, T., Toba, K., Yoshida, K., et al. (2010). Lipocalin-2/neutrophil gelatinase-B associated lipocalin is strongly induced in hearts of rats with autoimmune myocarditis and in human myocarditis. Circulation journal : official journal of the Japanese Circulation Society 74, 523530.

do Val, M. L., Menezes, F. S., Massaoka, H. T., Scavarda, V. T., Czapkowski, A., Leite, H. P., Moises, V. A., Ajzen, S. A., de Abreu Carvalhaes, J. T., Pestana, J. O. M., and Koch-Nogueira, P. (2019). Cardiovascular risk in children and adolescents with end stage renal disease. Clinics (Sao Paulo, Brazil) 74, e859.

Dolapoglu, A., and Beketaev, I. (2015). Can HbA1c and cholesterol levels affect the neutrophil gelatinaseassociated lipocalin (NGAL) after heart transplantation. Journal of cardiology 66, 269.

El-Gamasy, M. A., and Mawlana, W. H. (2019). Risk factors and prevalence of cardiac diseases in Egyptian pediatric patients with end-stage renal disease on regular hemodialysis. Saudi journal of kidney diseases and transplantation : an official publication of the Saudi Center for Organ Transplantation, Saudi Arabia 30, 5361.

Folkesson, M., Kazi, M., Zhu, C., Silveira, A., Hemdahl, A. L., Hamsten, A., Hedin, U., Swedenborg, J., and Eriksson, P. (2007). Presence of NGAL/MMP-9 complexes in human abdominal aortic aneurysms. Thrombosis and haemostasis 98, 427-433.

Hemdahl, A. L., Gabrielsen, A., Zhu, C., Eriksson, P., Hedin, U., Kastrup, J., Thoren, P., and Hansson, G. K. (2006). Expression of neutrophil gelatinaseassociated lipocalin in atherosclerosis and myocardial 
infarction. Arteriosclerosis, thrombosis, and vascular biology 26, 136-142.

Maisel, A. S., Mueller, C., Fitzgerald, R., Brikhan, R., Hiestand, B. C., Iqbal, N., Clopton, P., and van Veldhuisen, D. J. (2011). Prognostic utility of plasma neutrophil gelatinase-associated lipocalin in patients with acute heart failure: the NGAL EvaLuation Along with B-type NaTriuretic Peptide in acutely decompensated heart failure (GALLANT) trial. European journal of heart failure 13, 846-851.

Mitsnefes, M. M. (2012). Cardiovascular disease in children with chronic kidney disease. J Am Soc Nephrol 23, 578-585.

Niizuma, S., Iwanaga, Y., Yahata, T., and Miyazaki, S. (2017). Renocardiovascular Biomarkers: from the Perspective of Managing Chronic Kidney Disease and Cardiovascular Disease. Front Cardiovasc Med 4, 1010.

Park, M., Hsu, C.-Y., Go, A. S., Feldman, H. I., Xie, D., Zhang, X., Mifflin, T., Waikar, S. S., Sabbisetti, V. S., Bonventre, J. V., et al. (2017). Urine Kidney Injury Biomarkers and Risks of Cardiovascular Disease Events and All-Cause Death: The CRIC Study. Clin J Am Soc Nephrol 12, 761-771.

Poniatowski, B., Malyszko, J., BachorzewskaGajewska, H., Malyszko, J. S., and Dobrzycki, S. (2009). Serum neutrophil gelatinase-associated lipocalin as a marker of renal function in patients with chronic heart failure and coronary artery disease. Kidney \& blood pressure research 32, 77-80.

Shrestha, K., Borowski, A. G., Troughton, R. W., Thomas, J. D., Klein, A. L., and Tang, W. H. (2011). Renal dysfunction is a stronger determinant of systemic neutrophil gelatinase-associated lipocalin levels than myocardial dysfunction in systolic heart failure. Journal of cardiac failure 17, 472-478.

te Boekhorst, B. C., Bovens, S. M., Hellings, W. E., van der Kraak, P. H., van de Kolk, K. W., Vink, A., Moll, F. L., van Oosterhout, M. F., de Vries, J. P., Doevendans, P. A., et al. (2011). Molecular MRI of murine atherosclerotic plaque targeting NGAL: a protein associated with unstable human plaque characteristics. Cardiovascular research 89, 680-688.

Tomczak, J., Wasilewska, A., and Milewski, R. (2013). Urine NGAL and KIM-1 in children and adolescents with hyperuricemia. Pediatric nephrology (Berlin, Germany) 28, 1863-1869.

Yndestad, A., Landro, L., Ueland, T., Dahl, C. P., Flo, T. H., Vinge, L. E., Espevik, T., Froland, S. S., Husberg, C., Christensen, G., et al. (2009). Increased systemic and myocardial expression of neutrophil gelatinase-associated lipocalin in clinical and experimental heart failure. European heart journal 30, 1229-1236.

Zografos, T., Haliassos, A., Korovesis, S., Giazitzoglou, E., Voridis, E., and Katritsis, D. (2009). Association of neutrophil gelatinase-associated lipocalin with the severity of coronary artery disease. The American journal of cardiology 104, 917-920.

Zylka, A., Gala-Bladzinska, A., Dumnicka, P., Ceranowicz, P., Kuzniewski, M., Gil, K., Olszanecki, R., and Kusnierz-Cabala, B. (2016). Is Urinary NGAL Determination Useful for Monitoring Kidney Function and Assessment of Cardiovascular Disease? A 12-Month Observation of Patients with Type 2 Diabetes. Disease markers 2016, 8489543. 